\title{
Extracting More Capacity from Multi-Channel Multi- Radio Wireless Networks by Exploiting Power
}

\author{
Devu Manikantan Shila, Yu Cheng, Tricha Anjali \\ Department of Electrical and Computer Engineering \\ Illinois Institute of Technology, Chicago, IL
}

\author{
Peng-Jun Wan \\ Department of Computer Science \\ Illinois Institute of Technology, Chicago, IL
}

\begin{abstract}
Transmission power plays a crucial role in the design and performance of wireless networks. The issue is therefore complex since an increase in transmission power implies that a high quality signal is received at the receiver and hence an increase in channel capacity. Conversely, due to the shared nature of the wireless medium an increase in transmission power also implies high interference in the surrounding region and hence a quadratic reduction in the capacity of wireless networks. Recent literatures indicate that employing multiple channels can mitigate the negative effects of wireless interference and thus greatly improve the overall network capacity. Therefore, it is worth investigating the effect of exploiting power on the capacity of multi-channel multi-radio (MC-MR) wireless networks. Specifically, in this paper we address the following questions: (a) Can we maximize the capacity of MC-MR wireless networks by exploiting power? (b) Under what criteria can we increase the transmission power of the nodes in a MC-MR network?

When $n$ nodes each with $m$ half-duplex interfaces are optimally deployed in a torus of unit area, traffic patterns are optimally assigned, each transmission's range is optimally chosen and in the presence of $c$ channels, we show that in contrast to the setting where nodes transmit at minimum power level $P_{0}$ the transport capacity, measured in bit-meters per second, of MC-MR network exploiting power is increased by $\Theta\left(\frac{c}{c_{\min }}\right)$ in region $c_{\text {min }}<c<m n / 2$ and by $\Theta(n)$ in region $c \geq_{\alpha} m n / 2$ when nodes tune to transmit power level of $P_{0}\left(\frac{c}{c_{m i n}}\right)^{\frac{\alpha}{2}}$ and $P_{0} n^{\frac{\alpha}{2}}$ respectively-where $c_{\min }$ is the minimum number of channels required to achieve conflict-free transmissions in a network. Our analysis also sheds light into several insights that designers may want to consider to improve the performance of energy-efficient bandwidth-constrained wireless networks.
\end{abstract}

\section{INTRODUCTION}

In their seminal paper [1]-[2], Gupta and Kumar first derived the capacity of ad hoc wireless networks in the limit as the number of nodes grows to an arbitrarily large level. Under this model, the authors indicate that regardless of the initial location of the nodes and traffic pattern in a fixed area, the throughput in bit-meters/second cannot grow faster than $\sqrt{n}$. Consequently, the end-to-end throughput available for each node is $O(1 / \sqrt{n})$ which in turn approaches zero as the number of nodes $n$ increases. This pessimistic result stems from the fact that most communication has to occur between nearest neighbors, at distances of $O\left(\frac{1}{\sqrt{n}}\right)$, with each packet going through many other nodes (serving as relays) before reaching the destination. Therefore, it might appear that by reducing the number of hops traversed by each packet (i.e., by increasing the transmission power i.e., range) and thus the burden on nodes serving as relays, the throughput available to each node can be improved. However, the authors [2] point out that increasing transmission range (or power) may reduce the capacity due to spatial concurrency constraints and hence, one may really need to reduce the transmission power (or range) to as small a value as possible without sacrificing the connectivity.

Subsequently, on the assumption of minimal transmission power, a plethora of literatures were proposed to improve the capacity bounds of wireless networks by employing various techniques ranging from mobility [4], [5], [6] to multichannel multi-radio [7], UWB radios [9], [12], directional antennas [10], [11] etc. Moreover, since the power consumed by the radio frequency (RF) power amplifier of the network interface card (NIC) directly depends on the transmission power, there are a lot of research efforts [13]-[15], for e.g., COMPOW, that focus on designing power-optimal algorithms for maximizing the lifetime of the energy-constrained nodes such as sensor nodes. Surprisingly, there are opposite arguments recently [16]-[18]. In [16]-[18], researchers study the power problem by various approaches such as optimization, simulations etc and show that under some network configurations capacity can be indeed maximized by properly increasing the transmission power.

While the current state of art [2]-[6],[9]-[15] resort to utilizing minimum transmission power for maximizing spatial reuse, lifetime of energy-constrained nodes etc on single channel single-radio network (SC-SR) wireless network, we focus our study on the following aspects: (i) It is not understood yet the effect of employing power on multi-channel multiradio (MC-MR) wireless networks and hence, it is worth investigating whether we can extract more capacity from $\mathrm{MC}$ MR networks by exploiting power. This inspiration indeed comes from the fact that when the number of available channels $c$ is larger than $c_{\text {min }}$ (the minimum number of channels needed to achieve conflict-free transmissions in a network) i.e., $c>c_{\text {min }}$, the distance between co-channels can be enlarged by utilizing those extra channels $c-c_{\min }$. The larger cochannel distance also implies that each node can increase its transmission power without affecting the spatial reuse. (ii) As mentioned before, most studies focus on employing minimum power either to maximize spatial reuse [2]-[11] and/or lifetime of energy-constrained nodes [13]-[15] or to minimize the relaying burden on nodes [16]-[18]. However, the crucial parameter that these studies overlook is the signal quality at the receiver i.e., SNR (signal-to-noise ratio). Thus, we plan to exploit power to improve the SNR at the receiver.

According to Shannon-Hartley theorem [1], the capacity (bits/second) is a function of the SNR and hence an increase in SNR may be leveraged to increase the capacity which in turn can lead to a system with greater spectral efficiency. One may also note that a reduced transmission power as well as a larger 
distance between transmitter-receiver pair can in fact affect the quality of signal at the receiver and correspondingly the capacity. Therefore, in contrast to these existing research efforts we look at the power problem from a different perspective and are summarized as follows: (a) We exploit power to enhance the quality of the signal power at the receiver (i.e., SNR) without sacrificing the spatial reuse or lifetime of nodes; (b) As pointed out in [2], an increase in transmission power implies high interference in the surrounding region and consequently a quadratic reduction in the number of coexisting transmissions. As a result, we investigate the effect of transmission power on multi-channel multi-radio (MC-MR) wireless networks; and (c) Finally and most importantly, an increase in transmission power also implies increased energy consumption and hence a reduction in the lifetime of the nodes. Therefore, in this work we focus our attention on networks that have no power constraints such as Wireless Mesh Networks (WMNs) (see CellNode M100 [19]).

Motivated by these ideas, we specifically address the following two questions: (a) Can we maximize the capacity of MCMR wireless networks by increasing the transmission power? (b) Under what criteria can we increase the transmission power of the nodes in a MC-MR network? The results derived in this paper indicate that it may be possible to build capacity-optimal wireless networks by exploiting the transmission power in MC-MR network under the following two constraints: (i) $c_{\text {min }}=O(c)$ and $c=O(n m)$; (ii) $c=\Omega(n m)$-where $c_{\text {min }}$ is the minimum number of channels required to achieve conflict-free transmissions in the network and $c$ is the number of existing channels. Furthermore, surprisingly our results also point out that if the maximum transmit power is $\Omega(n)$ i.e., $P_{\max }=\Omega(n)$, then partitioning the fixed bandwidth into $\Theta(n m)$ subchannels leads to higher resource utilization and thus enhance the capacity of the wireless network. This indeed is an interesting and contrasting result to previously published works such as in [7].

In summary, the main contributions of this paper are the following:

- This paper produces the first effort to quantify the effect of employing power on the capacity of multi-radio wireless network operating on multiple channels.

- Since the key objective of this paper is to determine the optimal capacity region, we derive the upper and lower bounds on the capacity of the proposed model (MC-MR networks exploiting power) when node placements are chosen arbitrarily.

- Contrasting to existing literatures, we derive the bounds on capacity for the proposed model to show that unlike single channel wireless networks, MC-MR wireless networks in fact allow us to exploit power to obtain higher capacity and hence it may be possible to build capacityoptimal MC-MR networks by utilizing power.

- Furthermore, we show that the previously established bounds in [7] for multi-channel multi-radio (MC-MR) networks ${ }^{1}$ may not be accurate and thus presents necessary modifications to obtain more accurate results.

\footnotetext{
${ }^{1}$ We refer to MC-MR networks exploiting power as the proposed model and the MC-MR networks using minimum transmission power such as in [7] as the basic model.
}

\section{A. Definitions}

(a) Arbitrary network: We study the capacity of the proposed model under the arbitrary setting introduced by Gupta and Kumar [2]. In the arbitrary network setting, we suppose that $n$ nodes are arbitrarily located in a torus of unit area in the plane. Each node has an arbitrarily chosen destination to which it sends traffic at an arbitrary rate. Each node can chose an arbitrary range or power level for each transmission. Specifically, since the location of nodes, traffic pattern can be controlled in arbitrary setting, the bounds obtained for this scenario are applicable to any network and may be viewed as the best case bounds on network capacity especially for static multihop networks such as WMNs.

(b) Transport Capacity: We study the transport capacity of the network which is defined as the sum of the distances toward the destination traveled by every bit per unit time. Hence the transport capacity is measured in bit-meters per second. For instance, let $X_{R(k)}$ be the destination for the flow from node $X_{k}$. If $b_{k}(\tau)$ bits reach from node $X_{k}$ to receiver $X_{R(k)}$ in $\tau$ seconds, then $\sum_{k=1}^{n} b_{k}(\tau)\left\|X_{k}-X_{R(k)}\right\|$ bit-meters are transported in $\tau$ seconds, where $\|x-y\|$ denote the Euclidean distance between $x$ and $y$. The transport capacity of the network, $C_{T}$ is given by

$$
C_{T}=\lim _{\tau \rightarrow \infty} \frac{1}{\tau} \sum_{k=1}^{n} b_{k}(\tau)\left\|X_{k}-X_{R(k)}\right\|
$$

bit-meters per second.

\section{B. Notations}

We use the following asymptotic notation to represent the bounds:

- $f(n)=O(g(n))$ implies that $\exists k, N$, such that $f(n) \leq$ $k g(n)$ for $n>N$.

- $f(n)=o(g(n))$ implies that $\lim _{n \rightarrow \infty} \frac{f(n)}{g(n)}=0$.

- $f(n)=\omega(g(n))$ implies that $g(n)=o(f(n))$.

- $f(n)=\Omega(g(n))$ implies that $g(n)=O(f(n))$.

- $f(n)=\Theta(g(n))$ implies that $\exists k_{1}, k_{2}, N$, such that $k_{1} g(n) \leq f(n) \leq k_{2} g(n)$ for $n>N$.

The rest of the paper is organized as follows: Section II discusses the motivation behind this work. In section III, we initially start with the discussion of the network model and then focus on the models for communication. Section IV presents some useful lemmas used to obtain the capacity bounds of the proposed model and in section $\mathrm{V}$ we present the summary of our contributions. In section VI and VII, we establish the capacity of the proposed model under arbitrary settings. Section VIII presents the implications of the results derived in this paper. In section IX, we present the related work. Finally, we summarize our work in section X.

\section{Motivation}

In this section, we discuss an example to illustrate the power problem in MC-MR networks. For convenience of elucidation, here we consider a single radio multi-channel network as shown in Figure 1 and assume that each transmitterreceiver pair is placed at a distance of $O(1)$ apart. Suppose that $c$ channels are present in the network. Also, let $c_{\min }$ be the minimum number of channels needed to achieve the maximum 
number of simultaneous transmissions in a network $\left(\frac{n}{2}\right)$ when nodes transmit at the minimum power level say $P_{0}$. Let us also assume that $c_{\min }<c$.

In the Figure 1(left), we assign each overlapping interference disk with a distinct channel. Since all transmitters in this setting employ power level $P_{0}$, the number of channels needed to achieve the maximum simultaneous transmissions is $c_{\text {min }}$. Recall that $c>c_{\min }$ channels are present in the network. It should be pretty obvious from the Figure 1(left) that there is no benefit in assigning those remaining $c-c_{\min }$ channels to this particular setting since the network has already reached the maximum number of simultaneous transmissions. Thus the question arise, how could we exploit the residual $c-c_{\min }$ channels? What if we allow each transmitter to increase its power from $P_{0}$ to say $P>P_{0}$ such that all the channelsthat is $c$ - present in the network are completely utilized. We show this scenario in Figure 1(right) where each node increase its transmission power from $P_{0}$ to the power $P$ where all the existing $c$ channels are exploited. ${ }^{2}$
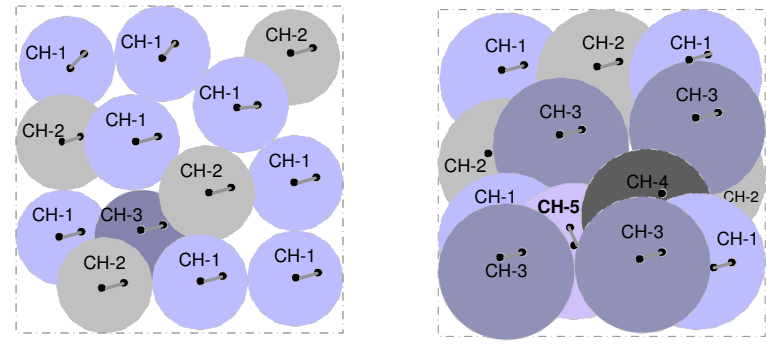

Fig. 1. Figure on the left shows transmitter-receiver pairs transmit at a minimum power level $P_{0}$ and utilizing $c_{\min }$ channels. While figure on the right shows the same setting when nodes transmit at a power level $P>P_{0}$ and utilizing $c$ channels. Each circle (or disk) corresponds to the interference disk around a transmitter-receiver pair.

Next we determine the transport capacity, measured in bitsmeters/sec, of the multi-channel network transmitting at power levels $P_{0}$ and $P>P_{0}$. Let $B_{P_{0}}$ and $B_{P}$ be the bit rate between each transmitter-receiver pair employing power levels $P_{0}$ and $P$ respectively. Since each overlapping disk is assigned a distinct channel under both settings, we have $\frac{n}{2}$ simultaneous transmissions and hence the network capacity for transmit power level $P_{0}$ and $P$ are $B_{P_{0}} \frac{n}{2}$ and $B_{P} \frac{n}{2}$ respectively. Based on Shannon-Hartley theorem, we know that an increase in transmission power implies high data rate and hence we have $B_{P} \frac{n}{2}>B_{P_{0}} \frac{n}{2}$. As this example indicates, when the available number of channels $c$ is larger than $c_{\text {min }}$, we can still extract more capacity from multi-channel network by exploiting power. This indeed is the motivation behind this work.

\section{BACKGROUND}

The objective of this paper is to derive the bounds on the capacity of the proposed model when node placements are chosen arbitrarily. For the ease of exposition, we start with the discussion of the network model and then focus on the models for communication.

\footnotetext{
${ }^{2}$ note that an increase in transmission power implies an increase in interference disk.
}

\section{A. Network Model and Assumptions}

Consider a network of $n$ nodes in a torus of unit area. Let $X_{i}, 1 \leq i \leq n$, denote the location of node $i$. We will use $X_{i}$ to denote a node as well as its location. Let $\left\{\left(X_{i}, X_{R(i)}\right): i \in T_{X}\right\}$ be the set of all transmitter-receiver pairs in some particular slot and $P_{i}$ be the transmitted power levels for these pairs. Similar to [2], we also assume a slotted model for convenience of elucidation. Let the transmission radius and interference radius be denoted as $r(i)$ and $\operatorname{Ir}(i)$. We further take the following assumptions.

- We assume that there are $c$ channels in the network and each node is equipped with $m$ interfaces, $1 \leq m \leq c$. Moreover, we assume that all nodes transmit on an ideal channel without channel fading.

- We also assume that an interface is capable of transmitting or receiving data on any one channel at a given time.

- We assume that each node $X_{i}: i \in T_{X}$ is constrained to a maximum transmit power $P_{\max }$ such that $P_{0} \leq P_{i} \leq$ $P_{\max }$, where $P_{0}$ is the minimum transmit power.

\section{B. Impact of Power on Interference Model}

We study the capacity of the proposed model under the so-called model of communication, the protocol model. In this model, the transmission from node $X_{i}, i \in T_{X}$, is successfully received by the receiver $X_{R(i)}$ if and only if the receiving node $X_{R(i)}$ is in the transmission radius of the corresponding transmitting node $X_{i}$ and is out of the interference radius of all other transmitting nodes $X_{k}, k \in T_{X} \backslash i$. In [2], Gupta and Kumar do not explicitly take into account the dependency of power of each node on the interference under the protocol model. Thus, we modify the interference model by considering the power level of each node.

To derive the necessary and sufficient condition for a successful transmission, we first quantify the transmission and interference radius of a node in the wireless network as in [3].

1) Transmission and Interference Radii: From the theory of communication, we know that both the transmission and interference radius of a node in fact depends on two factorstransmission power and the propagation gain. Thus, for a transmission from node $X_{i}$ to its receiver $X_{R(i)}$, we employ the following widely used model for propagation gain, $g_{i, R(i)}=\left(\left\|X_{i}-X_{R(i)}\right\|\right)^{-\alpha}$, where $\alpha>2$ is the path loss exponent and $\left\|X_{i}-X_{R(i)}\right\|$ is the physical distance between transmitter-receiver pair $X_{i}$ and $X_{R(i)}$. In this context, we assume that a data transmission from node $X_{i}$ to receiver $X_{R(i)}$ is successful only if the received signal strength at $X_{R(i)}$ exceeds a power threshold, say $\eta$ i.e., $\frac{P_{i}}{\left(\left\|X_{i}-X_{R(i)}\right\|\right)^{\alpha}} \geq \eta$. Then, the transmission radius of the node, denoted as $r(i)$ is:

$$
r(i)=\left(\frac{P_{i}}{\eta}\right)^{1 / \alpha}
$$

Similarly, we assume that a transmission from node $X_{i}$ is successfully received at $X_{R(i)}$ only if the interference power level does not exceed a threshold, say $\beta$ at the receiver. Following the same derivation for the transmission radius, the interference radius of a node, denoted as $\operatorname{Ir}(i)$ is obtained as follows:

$$
\operatorname{Ir}(i)=\left(\frac{P_{i}}{\beta}\right)^{1 / \alpha}
$$


2) Necessary and Sufficient Condition for Successful Transmission: For a given channel $x \in c$, we present the necessary and sufficient conditions to schedule a successful transmission from node $X_{i}$ to its receiver node $X_{R(i)}$ under the protocol model.

1) The receiving node $X_{R(i)}$ must be physically within the transmission radius of node $X_{i}$ i.e.,

$$
\left\|X_{i}-X_{R(i)}\right\| \leq r(i)=\left(\frac{P_{i}}{\eta}\right)^{1 / \alpha}
$$

2) The receiving node $X_{R(i)}$ should lie outside the interference radius of any other node $k \in T_{X} \backslash i$ that is transmitting in the same channel, i.e.,

$$
\left\|X_{k}-X_{R(i)}\right\| \geq \operatorname{Ir}(k)=\left(\frac{P_{k}}{\beta}\right)^{1 / \alpha}
$$

\section{General Physical Model}

We use Shannon's capacity formula for the additive white Gaussian noise channel to model the data rate. In this model, the data rate is a function of the signal-to-interference-plusnoise ratio (SINR) at the receiver. In this case the data rate from transmitter $X_{i}$ to its receiver $X_{R(i)}$ is given in bits/sec by

$$
B_{i}=W_{x} \log _{2}\left(1+\frac{\frac{P_{i}}{\left(\left\|X_{i}-X_{R(i)}\right\|\right)^{\alpha}}}{N_{0} W_{x}+\sum_{k \in T_{X}, k \neq i} \frac{P_{k}}{\left(\left\|X_{k}-X_{R(i)}\right\|\right)^{\alpha}}}\right)
$$

where $W_{x}$ is the bandwidth of the channel $x \in c$ in hertz, and $\frac{N_{0}}{2}$ is the noise spectral density in watts/hertz.

However, recall that we employ protocol model to characterize the interference and hence under the so-called protocol model for each receiver $X_{R(i)}$ if all the transmitters $X_{k} \in T_{X} \backslash i$ are more than $\operatorname{Ir}(k)$ distance away, then the accumulated interference at $X_{R(i)}$ will be negligible.

Hence, equation 3 can be rewritten as

$$
B_{i}=W_{x} \log _{2}\left(1+\frac{\frac{P_{i}}{\left(\left\|X_{i}-X_{R(i)}\right\|\right)^{\alpha}}}{N_{0} W_{x}}\right)
$$

Moreover, recall that in subsection III-A, we assume that each node is constrained to a maximum transmit power $P_{\max }$ such that $P_{0} \leq P_{i} \leq P_{\max }$, where $P_{0}$ is the minimum transmit power. For the simplicity of analysis, let minimum $\left(P_{0}\right)$ and maximum $\left(P_{\max }\right)$ transmit power level be $N_{0} W_{x} \eta\left\|X_{i}-X_{R(i)}\right\|^{\alpha}$ and $P_{0} n^{\frac{\alpha}{2}}=$ $N_{0} W_{x} \eta\left(\sqrt{n}\left\|X_{i}-X_{R(i)}\right\|\right)^{\alpha}$ respectively in the sequel.

\section{Assumptions on Channel Model}

As in [7], we consider the following basic assumptions on the channel model.

- Channel Model A: In this model, we have $W_{x}=\frac{W}{c}$ for all $x \in c$. Intuitively, we can see that as the number of channels $(c)$ increases the bandwidth for each channel decreases and hence the data rate supported by each channel will be less (see eq (4)). This model is applicable to scenarios where the total available bandwidth is fixed and new channels are created by splitting the existing channels.
- Channel Model B: In this model, we have $W_{x}=W$ for all $x \in c$. Intuitively, we can observe that each channel has a bandwidth of $W$ and hence, this model is applicable to scenarios where new channels are created by utilizing additional frequency spectrum.

\section{Some Useful Results}

In this section, we derive some results that is used to obtain the upper bound of the proposed model.

Lemma 1. Receiver-Based Interference Model: In a wireless network under protocol model, let $\left(X_{i}, X_{R(i)}\right)$ and $\left(X_{k}, X_{R(k)}\right)$ be two active transmitter-receiver pairs, then the disks of radius $\frac{\left(\frac{k_{3} \eta}{\beta}\right)^{\frac{1}{\alpha}}-1}{2}\left(\left\|X_{i}-X_{R(i)}\right\|+\left\|X_{k}-X_{R(k)}\right\|\right)$ centered at receivers over the same channel in the same slot are essentially disjoint.

Proof: Let $\left(X_{i}, X_{R(i)}\right)$ and $\left(X_{k}, X_{R(k)}\right)$ be two active transmitter-receiver pairs. From equation 1 , we can compute $P_{i}$ and $P_{k}$ respectively as follows:

$$
\begin{gathered}
P_{i} \geq \eta\left\|X_{i}-X_{R(i)}\right\|^{\alpha}=k_{3} \eta\left\|X_{i}-X_{R(i)}\right\|^{\alpha} \\
P_{k} \geq \eta\left\|X_{k}-X_{R(k)}\right\|^{\alpha}=k_{3} \eta\left\|X_{k}-X_{R(k)}\right\|^{\alpha}
\end{gathered}
$$

where $k_{3}$ is a constant and it takes values $N_{0} W_{x}$ for power level $P_{0}$ and $N_{0} W_{x} n^{\frac{\alpha}{2}}$ for power level $P_{\max }$.

Recall that the protocol model places the following constraints on the relative locations of these nodes and using equation 2, we get:

$$
\begin{array}{r}
\left\|X_{k}-X_{R(i)}\right\| \geq\left(\frac{k_{3} \eta}{\beta}\right)^{\frac{1}{\alpha}}\left\|X_{k}-X_{R(k)}\right\| \\
\left\|X_{i}-X_{R(k)}\right\| \geq\left(\frac{k_{3} \eta}{\beta}\right)^{\frac{1}{\alpha}}\left\|X_{i}-X_{R(i)}\right\|
\end{array}
$$

Using the triangle inequality first, we can derive the following relation between the location of the node pairs $\left(X_{i}, X_{R(i)}\right)$ and $\left(X_{k}, X_{R(k)}\right)$.

$$
\begin{array}{r}
\left\|X_{R(i)}-X_{R(k)}\right\|+\left\|X_{k}-X_{R(k)}\right\| \geq\left\|X_{R(i)}-X_{k}\right\| \\
\left\|X_{R(i)}-X_{R(k)}\right\| \geq\left(\frac{k_{3} \eta}{\beta}\right)^{\frac{1}{\alpha}}\left\|X_{k}-X_{R(k)}\right\|-\left\|X_{k}-X_{R(k)}\right\|
\end{array}
$$

Similarly we can write,

$$
\left\|X_{R(i)}-X_{R(k)}\right\| \geq\left(\frac{k_{3} \eta}{\beta}\right)^{\frac{1}{\alpha}}\left\|X_{i}-X_{R(i)}\right\|-\left\|X_{i}-X_{R(i)}\right\|
$$

Adding the inequalities in (7) and (8), we get

$$
\left\|X_{R(i)}-X_{R(k)}\right\| \geq \frac{\left(\frac{k_{3} \eta}{\beta}\right)^{\frac{1}{\alpha}}-1}{2}\left(\left\|X_{i}-X_{R(i)}\right\|+\left\|X_{k}-X_{R(k)}\right\|\right)
$$

Note that $X_{R(i)}$ and $X_{R(k)}$ are the receivers and we can deduce this inequality to say that for transmitter-receiver pairs $\left(X_{i}, X_{R(i)}\right)$ and $\left(X_{k}, X_{R(k)}\right)$ to be active, then a disk of radius $\frac{\left(\frac{k_{3} \eta}{\beta}\right)^{\frac{1}{\alpha}}-1}{2}\left(\left\|X_{i}-X_{R(i)}\right\|\right)$ centered at $X_{R(i)}$ and a disk of radius $\frac{\left(\frac{k_{3} \eta}{\beta}\right)^{\frac{1}{\alpha}}-1}{2}\left(\left\|X_{k}-X_{R(k)}\right\|\right)$ centered at $X_{R(k)}$ should not overlap. We term this model as the receiver-based interference model. 
Lemma 2. Sender-Based Interference Model: In a wireless network under protocol model, let $\left(X_{i}, X_{R(i)}\right)$ and $\left(X_{k}, X_{R(k)}\right)$ be two active transmitter-receiver pairs, then the disks of radius $\left(1+\frac{\left(\frac{k_{3} \eta}{\beta}\right)^{\frac{1}{\alpha}}-1}{2}\right)\left(\left\|X_{i}-X_{R(i)}\right\|+\right.$ $\left.\left\|X_{k}-X_{R(k)}\right\|\right)$ centered at transmitters over the same channel in the same slot are essentially disjoint.

Proof: This lemma can be easily proved from the previous Lemma 1 and hence the proof is omitted here for brevity.

The receiver-based and sender-based interference models are indeed the same. While the former focuses on the interference region around the receiver, the latter focuses on the transmitter. In the sequel, we adopt the sender-based interference model to develop the capacity bounds of the proposed model. However, note that all the results in this paper hold true under both interference models.

Lemma 3. The number of simultaneous transmissions on any particular channel is no more than

$$
\frac{1}{\pi\left(1+\frac{\Delta}{2}\right)^{2} d^{2}}
$$

where $\Delta=\left(\frac{k_{3} \eta}{\beta}\right)^{\frac{1}{\alpha}}-1$.

Proof: Let $\left\|X_{i}-X_{R(i)}\right\|=d$ for all $i \in T_{X}$. Hence, according to Lemma 2, disks of radius $\left(1+\frac{\Delta}{2}\right) d$ centered at each transmitter on the channel $x \in c$ are essentially disjoint. Since the area of each such disk is $\pi\left(1+\frac{\Delta}{2}\right)^{2} d^{2}$, it follows that the network can support no more than $\frac{1}{\pi\left(1+\frac{\Delta}{2}\right)^{2} d^{2}}$ simultaneous transmissions on any channel $x \in c$.

Lemma 4. Suppose each node transmits at a power level $P_{0}$, then the minimum number of channels, $c_{\text {min }}$, required to achieve conflict-free transmissions in the network is no more than

$$
\frac{k_{4} \pi\left(1+\frac{\Delta}{2}\right)^{2} d^{2} n m}{2}
$$

where $\Delta=\left(\frac{k_{3} \eta}{\beta}\right)^{\frac{1}{\alpha}}-1$ and $k_{3}=N_{0} W_{x}$ for power level $P_{0}$.

Proof: Since each node has $m$ interfaces, there are total of $n m$ interfaces in the network. Noting that each interface cannot transmit and receive at the same time, the maximum number of pairs of interfaces available for simultaneous transmission is no more than $m n / 2$. Lemma 3 states that the number of simultaneous transmissions on any particular channel is no more than $\frac{1}{\pi\left(1+\frac{\Delta}{2}\right)^{2} d^{2}}$ and therefore, to achieve $\mathrm{nm} / 2$ simultaneous transmissions, the minimum number of channels $c_{\min }$ cannot be more than

$$
\begin{aligned}
& \frac{c_{\min }}{\pi\left(1+\frac{\Delta}{2}\right)^{2} d^{2}} \geq \frac{n m}{2} \\
c_{\text {min }}= & \frac{k_{4} \pi\left(1+\frac{\Delta}{2}\right)^{2} d^{2} n m}{2}
\end{aligned}
$$

where $k_{4}$ is a constant.

Clearly, we can deduce from Lemmas (1)-(4) that an increase in power implies a linear increase in disk radius which in turn implies a quadratic increase in minimum number of channels, $c_{\text {min }} \propto \Delta^{2}=\left(\left(\frac{k_{3} \eta}{\beta}\right)^{\frac{1}{\alpha}}-1\right)^{2}$, required to achieve interference-free transmissions in the network.
Lemma 5. For $z \in R^{+}$and $a \geq 1, \ln \left(1+z^{a}\right) \leq a z$

Proof:

$$
\begin{aligned}
\ln \left(1+z^{a}\right) & \leq \ln \left((1+z)^{a}\right) \\
& =a \ln (1+z) \leq a z
\end{aligned}
$$

\section{MAin Result}

In this section, we present the capacity bounds of MCMR networks exploiting power for the following scenarios: (a) $c_{\text {min }}<c<n m / 2$; (b) $c_{\text {min }} \geq c$ and (c) $c \geq n m / 2$.

Under the sender-based interference model and when node placements are chosen arbitrarily, the transport capacity of the proposed model measured in bit-meters/second under channel models A and B is given by:

Channel Model A:

$$
:= \begin{cases}\Theta\left(W \sqrt{\frac{n m}{c_{m i n}}}\right) & \text { when } c_{\text {min }}<c<n m / 2 ; \\ \Theta\left(W \sqrt{\frac{n m}{c}}\right) & \text { when } c_{\text {min }} \geq c ; \\ \Theta\left(\frac{W n^{2} m}{c}\right) & c \geq n m / 2 ;\end{cases}
$$

Channel Model B:

$$
:= \begin{cases}\Theta\left(W c \sqrt{\frac{n m}{c_{m i n}}}\right) & \text { when } c_{\text {min }}<c<n m / 2 ; \\ \Theta(W \sqrt{n m c}) & \text { when } c_{\text {min }} \geq c ; \\ \Theta\left(W n^{2} m\right) & c \geq n m / 2 ;\end{cases}
$$

\section{AN UPPER BOUND ON TRANSPORT CAPACITY}

We consider $n$ nodes arbitrarily located in a torus of unit area on the plane. The following are the basic assumptions on the model being considered:

1) The network transports $\lambda n T$ bits over $T$ seconds.

2) The average distance between the source and destination of a bit is $\bar{L}$. Together with (1), this implies that a bitmeters/second of $\lambda n \bar{L}$ is achieved.

3) Transmissions are slotted into synchronized slots of length $\tau$ seconds.

4) There are $c$ channels present in the network and each node has $m(1 \leq m \leq c)$ half-duplex interfaces (or radios). Moreover, we assume that each node in the network employ the same transmit power in all channels. This model is referred to as Symmetric Allocation. In Appendix, we also analyze a different setting where all nodes do not employ the same transmit power which is referred to as Asymmetric Allocation.

5) There also exists minimum number of channels $c_{\min } \leq$ $c$ or $c_{\min } \geq c$ with which the network can achieve interference-free transmissions.

6) When the domain is of $A$ square meters rather than 1 square meters, then all the upper bounds are scaled by $\sqrt{A}$.

Theorem 1. Consider a wireless network with $n$ nodes each with $m$ half-duplex interfaces deployed in a domain of unit area under the sender-based interference model, then the transport capacity $\lambda n \bar{L}$ is upper bounded as follows:

Channel Model A: 
- when $c_{\min }<c<n m / 2$

$$
\lambda n T \bar{L} \leq \frac{\sqrt{2} k_{5} W T}{(2+\Delta)} \sqrt{\frac{n m}{\pi c_{\min }}} \text { bit }- \text { meters/second }
$$

- when $c_{\min } \geq c$

$$
\lambda n T \bar{L} \leq \frac{\sqrt{2} k_{6} W T}{(2+\Delta)} \sqrt{\frac{n m}{\pi c}} \text { bit - meters/second }
$$

\section{Channel Model B:}

- when $c_{\min }<c<n m / 2$

$$
\lambda n T \bar{L} \leq \frac{\sqrt{2} k_{6} W T c}{(2+\Delta)} \sqrt{\frac{n m}{\pi c_{\text {min }}}} \text { bit - meters/second }
$$

- when $c_{\min } \geq c$

$$
\lambda n T \bar{L} \leq \frac{\sqrt{2} k_{6} W T}{(2+\Delta)} \sqrt{\frac{n m c}{\pi}} \text { bit - meters/second }
$$

where $k_{5}=\left(\eta^{\frac{2}{\alpha}} \frac{\alpha}{2}\right) /(\ln 2)$ and $k_{6}=\eta /(\ln 2)$.

Proof: Consider an arbitrary bit $b$, where $1 \leq b \leq \lambda n T$. Let the number of hops that bit $b$ traverses from its origin to its destination in a sequence of $h(b)$ hops, where the $h$ th hop traverses a distance of $d_{b}^{h}$. Then from assumption (2), we have

$$
\sum_{b=1}^{\lambda n T} \sum_{h=1}^{h(b)} d_{b}^{h} \geq \lambda n T \bar{L}
$$

which follows from the fact that the line segment has the shortest length among all curves passing two given points.

Let $H$ be the total number of hops traversed by all bits in $T$ seconds i.e., $H=\sum_{b=1}^{\lambda n T} h(b)$. Since each node has $m$ interfaces, and each interface transmits over a channel with rate $B$ bits per second ${ }^{3}$, the total number of bits that can be transmitted by all nodes over all interfaces is at most $\frac{B T n m}{2}$ (where the factor $1 / 2$ is based on the half-duplex nature of the interface). Hence, we have

$$
H=\sum_{b=1}^{\lambda n T} h(b) \leq \frac{B T n m}{2}
$$

From Lemma (2), we know that a transmission over a hop of length $d$ is successful only is there is no transmitter within a distance of $(2+\Delta) d$, where $\Delta=\left(\frac{k_{3} \eta}{\beta}\right)^{\frac{1}{\alpha}}-1$. Alternatively, we can say that two disks of radius $\left(1+\frac{\Delta}{2}\right) d$ centered around the transmitters are essentially disjoint. This may be viewed as each hop consuming a disk of radius $\left(1+\frac{\Delta}{2}\right)$ times the length of the hop around each transmitter. Since the area consumed on each channel is bounded above by the area of the domain $\left(=1 \mathrm{~m}^{2}\right)$, summing over all channels and the slots (there can be no more than $\frac{T}{\tau}$ slots in $T$ seconds) we have the following constraint:

Remark 1. We are particularly interested in the scenario where $c_{\min }<c<m n / 2$. In [7], the authors assume that $c$ channels are present in the network and hence the summation of area consumed on each channel, $x \in c$, sum to 1 . However, this reasoning is not accurate. In Lemma 4, we see that there also exists a minimum number of channels, $c_{\min }$ where

\footnotetext{
${ }^{3}$ Since nodes employ same power (see assumption (4)), we assume that each node transmit over the channel with same data rate $B$ i.e., $B_{i}=B \forall i \in T_{X}$
}

$c_{\text {min }}<c$ or $c_{\text {min }}>c$, with which the network can achieve conflict-free transmissions. Hence $c_{\text {min }}<c$ implies that the area consumed on each channel is upper bounded by $O\left(\frac{c_{\min }}{c}\right)$. This also implies that if each node increases its disk radius by $\sqrt{\frac{c}{c_{\text {min }}}}$, then all existing $c$ channels can be exploited. On the other hand, note that the reasoning of [7] holds true when $c_{\text {min }} \geq c$.

$$
\sum_{b=1}^{\lambda n T} \sum_{h=1}^{h(b)} \pi\left(1+\frac{\Delta}{2}\right)^{2}\left(d_{b}^{h}\right)^{2} \leq B T \min \left(c_{\min }, c\right)
$$

which can be rewritten as

$$
\sum_{b=1}^{\lambda n T} \sum_{h=1}^{h(b)} \frac{1}{H}\left(d_{b}^{h}\right)^{2} \leq \frac{4 B T \min \left(c_{\min }, c\right)}{\pi(2+\Delta)^{2}}
$$

Noting that the quadratic equation is convex, we have

$$
\left(\sum_{b=1}^{\lambda n T} \sum_{h=1}^{h(b)} \frac{1}{H} d_{b}^{h}\right)^{2} \leq \sum_{b=1}^{\lambda n T} \sum_{h=1}^{h(b)} \frac{1}{H}\left(d_{b}^{h}\right)^{2}
$$

Combining (12) and (13) yields

$$
\sum_{b=1}^{\lambda n T} \sum_{h=1}^{h(b)} d_{b}^{h} \leq \sqrt{\frac{4 B T H \min \left(c_{\min }, c\right)}{\pi(2+\Delta)^{2}}}
$$

Now substituting (9) and (10) in (14) yields the result

$$
\lambda n T \bar{L} \leq \frac{\sqrt{2} B T}{(2+\Delta)} \sqrt{\frac{n m \min \left(c_{\min }, c\right)}{\pi}}
$$

To get the final capacity bounds, we need to consider the following scenarios where different values for data rate $B$ are obtained.

CASE A: $c_{\min }<c<m n / 2$. Recall that while $c$ channels are present in the network, no more than $c_{\min }$ channels are utilized. Hence to completely exploit the existing $c$ channels, each node can increase the disk size by $\sqrt{\frac{c}{c_{m i n}}}$ which in turn implies that each node can augment the power $P$ from $P_{0}$ to $P_{0}\left(\sqrt{\frac{c}{c_{\min }}}\right)^{\alpha}$. Substituting $P_{0}\left(\sqrt{\frac{c}{c_{\min }}}\right)^{\alpha}$ in equation (4) and using Lemma 5, we get ${ }^{4}$

$$
\begin{aligned}
B & =W_{x} \log _{2}\left(1+\eta\left(\sqrt{\frac{c}{c_{\text {min }}}}\right)^{\alpha}\right)=W_{x} \log _{2}\left(1+\left(\eta^{\frac{2}{\alpha}} \frac{c}{c_{\text {min }}}\right)^{\frac{\alpha}{2}}\right) \\
& =\frac{W_{x}}{\ln (2)} \ln \left(1+\left(\eta^{\frac{2}{\alpha}} \frac{c}{c_{\min }}\right)^{\frac{\alpha}{2}}\right) \\
& \leq \frac{W_{x}}{\ln (2)} \eta^{\frac{2}{\alpha}} \frac{\alpha}{2}\left(\frac{c}{c_{\text {min }}}\right)=W_{x} k_{5} \frac{c}{c_{\text {min }}}
\end{aligned}
$$

where $k_{5}=\left(\eta^{\frac{2}{\alpha}} \frac{\alpha}{2}\right) /(\ln 2)$. Since $\frac{\alpha}{2} \geq 1$, we can use Lemma 5 to replace $\ln (1+x)^{\frac{\alpha}{2}}$ by $(\alpha / 2) x$.

Substituting $B=W_{x} k_{5} \frac{c}{c_{m i n}}$ in (15) and $W_{x}=W / c$, we get

$$
\lambda n T \bar{L} \leq \frac{\sqrt{2} k_{5} W T}{(2+\Delta)} \sqrt{\frac{n m}{\pi c_{\min }}}
$$

CASE B: $c_{\min } \geq c$. In this setting when minimum number of channels, $c_{m i n}$, is greater than the existing channels i.e.,

\footnotetext{
${ }^{4}$ we used the following $\log _{2}(x)=\frac{\log _{e}(x)}{\log _{e}(2)}$ to replace $\log _{2}(x)$ by $\log _{e}(x)$.
} 
$\min \left(c_{\min }, c\right)=c$, each node can transmit only at the minimum power level $P_{0}$. Hence substituting $P_{0}$ in equation (4) and using Lemma 5 , we get $B=\frac{W_{x}}{\ln 2} \eta$. Now, combining $B=\frac{W_{x}}{\ln 2} \eta$ and $W_{x}=W / c$ in 15 , we obtain

$$
\lambda n T \bar{L} \leq \frac{\sqrt{2} k_{6} W T}{(2+\Delta)} \sqrt{\frac{n m}{\pi c}}
$$

where $k_{6}=\eta /(\ln 2)$.

Remark 2. We can see that in the scenario where $c_{\min }<c<$ $\mathrm{mn} / 2$, the capacity of the arbitrary network under minimum power level $P_{0}$ (i.e., basic model), is

$$
\lambda n T \bar{L} \leq \frac{\sqrt{2} k_{6} W T}{(2+\Delta) c} \sqrt{\frac{n m c_{\min }}{\pi}}
$$

Clearly, we can observe that ignoring the constants the proposed model has a gain of $\frac{c}{c_{\text {min }}}$ over the basic model under the constraint $c_{\min }<c<m n / 2$.

The derivation of the proof is based on the assumption of channel model A. However, all the results under channel model B can be obtained by replacing $W$ by $W c$.

Theorem 2. Consider a wireless network with $n$ nodes each with $m$ half-duplex interfaces deployed in a domain of unit area and when $c \geq m n / 2$, then the transport capacity is upper bounded as follows:

\section{- Channel Model A}

$$
\lambda n T \bar{L} \leq \frac{W k_{5} n^{2} m}{2 c} \text { bit }- \text { meters } / \text { second }
$$

- Channel Model B

$$
\lambda n T \bar{L} \leq \frac{W k_{5} n^{2} m}{2} \text { bit }- \text { meters } / \text { second }
$$

where $k_{5}=\left(\eta^{\frac{2}{\alpha}} \frac{\alpha}{2}\right) /(\ln 2)$.

Proof: The capacity of arbitrary networks is also constrained by the maximum number of bits that can be transmitted simultaneously over all interfaces in the network. Since each node has $m$ interfaces, there are total of $n m$ interfaces in the network. Each interface can transmit at a data rate of $B$ bits per second. Also, the maximum distance a bit can travel in the network is $O(1)$ meters. Hence, the total network capacity is at most $O\left(B \frac{n m}{2}\right)$ bit-meters/sec.

However, noting that the maximum number of simultaneous transmissions feasible in this network is $n \mathrm{~m} / 2$ and hence when $c \geq n m / 2$ channels are present, indeed each node can tune its interface to a different channel. This as well as implies that each node can transmit to a maximum power level, $P_{\max }=P_{0}(\sqrt{n})^{\alpha}$, without causing interference to other transmissions in the network. Therefore using $P_{\max }$ in equation (4) and employing Lemma 5, we get $B=W_{x} k_{5} n$. Now, combining $B=W_{x} k_{5} n$ and $W_{x}=W / c$ in $O\left(B \frac{n m}{2}\right)$, the network capacity is obtained as $O\left(\frac{W k_{5} n^{2} m}{2 c}\right)$.

Remark 3. We can see that in the scenario where $c \geq m n / 2$, the capacity of the arbitrary network under minimum power level $P_{0}$ (i.e., basic model), is $O\left(\frac{W k_{6} n m}{2 c}\right)$. Indeed, we can observe that ignoring the constants the proposed model has a gain of $n$ over the basic model under the constraint $c \geq m n / 2$.

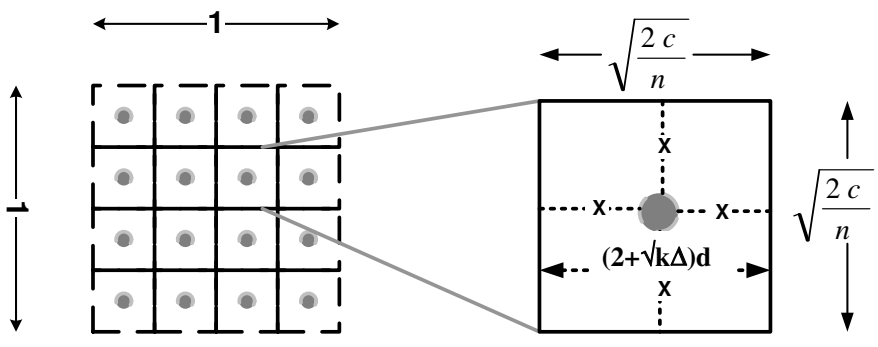

Fig. 2. Figure on the left shows the arrangement of the transmitters in the domain of area $=1 m^{2}$. The distance between every transmitter is $(2+\sqrt{k} \Delta) d$ and hence, according to the sender-based interference model the disk of radius $\left(1+\sqrt{k} \frac{\Delta}{2}\right) d$ around each transmitter do not overlap. Figure on the right shows the enlarged view of the square cell. The receiver of the corresponding transmitter can be placed at any of the locations marked $X$ which is at a distance of $d$ from the transmitter.

\section{A LOWER BOUND ON TRANSPORT CAPACITY}

We will now manifest that the upper bound obtained in the previous section is indeed sharp under the senderbased interference model by exhibiting a scenario where it is achieved. Also, note that in this section we provide the lower bound construction for a single interface multi-channel network; however the results can be easily extended to a multiinterface network by using the Lemma from [7].

Theorem 3. When $c<n / 2$, there is a placement of nodes and an assignment of traffic patterns such that the network can achieve $\frac{W}{\Delta \sqrt{2}} \sqrt{\frac{n}{c_{m i n}}}$ bit-meters/sec for $\min \left(c_{\text {min }}, c\right)=c_{\text {min }}$ and $\frac{W}{\Delta \sqrt{2}} \sqrt{\frac{n}{c}}$ bit-meters/sec for $\min \left(c_{\min }, c\right)=c$ respectively under channel model $\mathrm{A}$.

Proof: We consider a torus of unit area. Partition the area into $\frac{n}{2 c}$ equal-sized square cells, and place $c$ transmitterreceiver pairs in each cell. Intuitively, this implies that transmitter-receiver pairs operating in distinct channels can be placed at the same location. The location of the transmitter in each cell is shown in Figure 2. Since the area of the domain is $1 m^{2}$, each cell has an area of $\frac{2 c}{n}$ and sides of length $s=\sqrt{\frac{2 c}{n}}$. Let $k=\frac{c}{\min \left(c_{\min }, c\right)}$. Each transmitter in a cell is placed at a distance of $(2+\sqrt{k} \Delta) d$ from the transmitter at its adjacent cell (recall the sender-based interference model). For instance, we can see that when $\min \left(c_{\min }, c\right)=c$, the distance between two adjacent transmitters is $(2+\Delta) d$ which corresponds to the diameter of the interference disk when nodes transmits at a power level $P_{0}$.

From the above construction, it can be verified that there are total of $\frac{n}{2 c} \times c=\frac{n}{2}$ simultaneous transmitter-receiver pairs located within the domain, each transmitting at a rate of $\frac{W}{c}(k)$ over a distance of $d=\frac{s}{(2+\sqrt{k} \Delta)}$. Hence the total capacity of the network is $\frac{W(k)}{c} \frac{n}{2} d=\frac{W(k)}{c} \frac{n}{2} \frac{s}{(2+\sqrt{k} \Delta)}$ bitmeters/sec. Recall that $k=\frac{c}{\min \left(c_{\min }, c\right)}$. Therefore substituting for $k$, we obtain the network capacity as $\approx \frac{W}{\Delta \sqrt{2}} \sqrt{\frac{n}{c_{\min }}}$ for $\min \left(c_{\min }, c\right)=c_{\min }$ and $\approx \frac{W}{\Delta \sqrt{2}} \sqrt{\frac{n}{c}}$ for $\min \left(c_{\min }, c\right)=c$.

Theorem 4. When $c \geq n / 2$, there is a placement of nodes and an assignment of traffic patterns such that the network can achieve $\frac{W n^{2}}{2 c}$ bit-meters/sec under channel model A. 

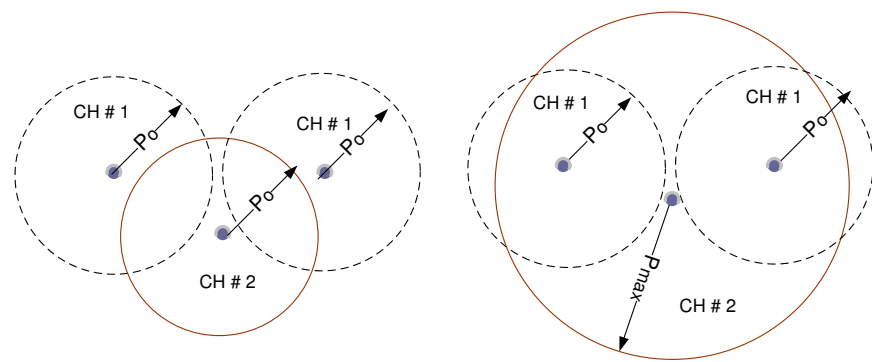

Fig. 3. Figure on the left shows that all transmitters transmit at a constant minimum power level $P_{0}$ while figure on the right shows the setting where some nodes can transmit at power level $>P_{0}$. Each circle corresponds to the interference disk around a transmitter-receiver pair.

Proof: Recall that the domain is a torus of unit area. Let $g=\min \left(c, \frac{n}{2}\right)$. Partition the area into $\frac{n}{2 g}$ cells, and place $g$ transmitter-receiver pairs in the cell. Intuitively, we can see that since $c \geq n / 2$, each $g$ transmitter-receiver pair will be operating on a distinct channel and hence each pair can be placed at the same location. Since the area of the domain is $1 m^{2}$, each cell has an area of $\frac{2 g}{n}$ and sides of length $s=\sqrt{\frac{2 g}{n}}$. Recall that $g=\min \left(c, \frac{n}{2}\right)$ and $c \geq n / 2$, hence length of the square cell is $s=1$. Each transmitter is placed at the center of the edge of the cell and its corresponding receiver is placed at the center of the opposite edge at a distance of $d$.

From the above construction, it can be verified that there are total of $\frac{n}{2 g} \times g=\frac{n}{2}$ simultaneous transmitter-receiver pairs located within the domain, each transmitting at a rate of $\frac{W}{c}\left(P_{\max }\right) \approx \frac{W n}{c}$ over a distance of $d=s=1$. Hence the total capacity of the network is $\frac{W n}{c} \frac{n}{2} d=\frac{W n^{2}}{2 c}$ bit-meters/sec

\section{DISCUSSIONS}

In Table I we study the gain of the MC-MR network exploiting power over the basic model under the constraint $c_{\text {min }}=O(c)$ and $c=O(n)$. Indeed, we can see that when the number of channels existing in the network, $c$, exceeds the minimum channels required for achieving conflict-free transmissions i.e., $c_{m i n}$, the proposed model has a gain of $\frac{c}{c_{m i n}}$ over the basic model. However, when $c_{\text {min }} \geq c$ we see that there is no point in increasing the transmit power level and consequently end with the same capacity as in [7]-i.e., gain $=\Theta(1)$. Therefore, one implication that follows from the results of this paper is to achieve a capacity gain of $\frac{c}{c_{m i n}}$, the designers should perhaps want to consider the constraint $c_{\min }<c$ while developing energy-efficient networks such as mesh networks.

TABLE I

Gain of the Proposed Model over Basic MOdel $\left(\frac{c}{c_{m i n}}\right)$ For CASE $m=1, c=O(n)$

\begin{tabular}{|c|ccc|}
\hline$c=O(n)$ & $c_{\min }=1$ & $c_{\min }=\log \log n$ & $c_{\min }=\log n$ \\
\hline \hline 1 & $\Theta(1)$ & $\Theta(1)$ & $\Theta(1)$ \\
$\log \log n$ & $\Theta(\log \log n)$ & $\Theta(1)$ & $\Theta(1)$ \\
$\log n$ & $\Theta(\log n)$ & $\Theta\left(\frac{\log n}{\log \log n}\right)$ & $\Theta(1)$ \\
\hline \hline
\end{tabular}

Yet another issue that arises is whether $\frac{c}{c_{\min }}$ is indeed the maximum gain achievable under the constraint $c_{\text {min }} \leq c$. For instance, consider the network of three transmitter-receiver pairs in Figure 3. Suppose that $c_{\min }=c=2$, then according to our approach each pair is constrained to a minimum transmit power level, $P_{0}$ and hence the network capacity in bits/second is $W_{x}\left(P_{0}\right) \frac{n}{2}$. However, we can see that even though $c_{\min }=c$, transmitter-receiver pair operating on channel 2 can indeed transmit at maximum power level $P_{\max }$ without causing interference to other transmissions; Thus, in this setting the network can achieve a capacity of $W_{x}\left(P_{0}\right) \frac{n-2}{2}+W_{x}\left(P_{\max }\right)$ bits/second which is obviously greater than the capacity at constant transmit power setting. Certainly, there is still room for capacity improvement and thus, we plan to focus on this topic for our future research work. Further, this may also offer some suggestive guidelines for designers of static mesh network.

Correspondingly in Table II, we study the capacity of both proposed and basic models for the case $m=1, c=\Omega(n)$. One interesting situation that arises from the result in Table II is that when $c=n$, even though the channels are partitioned into $c$ subchannels, the transport capacity of the network under the proposed model is $\Theta(W n)$ i.e, the end-to-end throughput available for each node is $\Theta(W)$. This is a contrasting result to [7] which claims that the end-to-end throughput of each node under channel partition approaches zero when $c \geq n$. This improvement can be elucidated regarding the data rateSNR (signal-to-noise ratio) relationship in Shannon-Hartley theorem. According to the theorem, the transmission rate i.e., the data rate per unit bandwidth is a function of SNR and hence an increase in SNR may be leveraged to increase the transmission rate. Therefore, while we employ the maximum power level $P_{\max }=P_{0} n^{\alpha / 2}$, the SNR in fact increases with the power i.e., SNR $\propto P_{\max }$ and thus we observe increased data rate for each partitioned channel. Alternatively, we can say that the bandwidth is efficiently utilized under the maximum transmit power level which in turn leads to a higher capacity at $c=n$. On the other hand, when $c>n$, the capacity of the arbitrary network indeed decreases with the channel partitioning. Therefore, one implication that follows from this result is that if the maximum transmit power is $\Omega(n)$ i.e., $P_{\max }=\Omega(n)$, then dividing the fixed bandwidth into $\Theta(n)$ subchannels does not degrade the capacity of the wireless network. This in fact is an interesting and novel result that the designers may perhaps want to consider while designing energy-efficient wireless networks.

TABLE II

CApacity of Basic Model and Proposed Model For Case $m=1, c=\Omega(n)$ AND UNDER CHANNEL MODEL A

\begin{tabular}{|c|cc|}
\hline$c=\Omega(n)$ & Basic Model & Proposed Model \\
\hline \hline$n$ & $\Theta(W)$ & $\Theta(W n)$ \\
$n \log n$ & $\Theta\left(\frac{W}{\log n}\right)$ & $\Theta\left(\frac{W n}{\log n}\right)$ \\
$n^{2}$ & $\Theta\left(\frac{W}{n}\right)$ & $\Theta(W)$ \\
\hline \hline
\end{tabular}

\section{RELATED WORK}

In this section, we initially focus our review on literatures that aim to improve the capacity of wireless networks in several ways and then present a few studies concentrated on implementing power control algorithms for wireless networks. 
In their landmark work [2], Gupta and Kumar first derived the capacity of ad hoc wireless networks in the limit as the number of nodes grows to an arbitrarily large level. Under this model, the authors indicate that regardless of the initial location of the nodes and traffic pattern in a fixed area, the throughput in bit-meters per second cannot grow faster than $\sqrt{n}$, and for a special arrangement of nodes and traffic pattern a throughput of $\sqrt{n}$ can be achieved. Therefore, the end-to-end throughput available for each node is $O(1 / \sqrt{n})$. Gupta and Kumar also demonstrated the existence of a global scheduling scheme achieving $\Omega(1 / \sqrt{n \log n})$ for a network with random traffic pattern and random node distribution. This pessimistic result that the end-to-end throughput available to each node approaches zero as the number of nodes $n$ increases motivated many researchers to improve the capacity bounds of wireless networks in several ways by employing mobility, directional antennas, UWB radios etc.

In [4], [5], [6], the results show that mobility can increase the capacity of wireless network. Under the assumption that nodes are mobile and the position of each node is ergodic with stationary uniform distribution on an open disk, Grossglauser and Tse [5] manifest that when nodes are mobile it is possible to have a constant or $O(1)$ throughput scaling per sourcedestination pair w.h.p at the cost of increased end-to-end delay as the number of nodes in each unit area goes to infinity. Consequently, several researchers analyzed the trade off between delay and capacity in mobile networks[5], [6]. In [7], the authors study the network capacity in the context where the number of interfaces $m$ at each node may be smaller than the number of available channels $c$. The result shows that the capacity results are a function of channel-to-interface $\frac{c}{m}$ ratio and under arbitrary network setting there are two distinct capacity regions: when $\frac{c}{m}$ is $O(n)$, the network capacity is $\Theta(W \sqrt{n m / c})$ and when $\frac{c}{m}$ is $\Omega(n)$, the network capacity is $\Theta(W n m / c)$.

In [8], Liu et. al employ the infrastructure support to improve the capacity bound. The work considers the scenario where $m$ base stations are placed in a regular hexagonal pattern within the ad hoc network with $n$ nodes and employs two routing strategies: deterministic and probabilistic. Under the deterministic routing strategy, the result shows that if $m$ grows asymptotically slower than $\sqrt{n}$, the maximum throughput capacity is $\Theta\left(\sqrt{n / \log \left(\frac{n}{m^{2}}\right)} W\right)$ and if $m$ grows faster than $\sqrt{n}$, the maximum capacity is $\Theta(m W)$, which increases linearly with the number of base stations. In the probabilistic routing strategy, if $m$ grows slower than $\sqrt{n / \log n}$, the maximum throughput capacity has the same asymptotic behavior as pure ad hoc network. If $m$ grows faster than $\sqrt{n / \log n}$, the maximum throughput capacity scales as $\Theta(m W)$, which increases linearly with the number of base stations.

Another relevant body of work is employing directional antennas to improve the capacity bounds under the context of single channel and multiple channel networks. Yi et. al [10] show that in a random wireless network, the use of directional antennas can improve the network capacity by a factor of $2 \pi / \alpha$, where $\alpha$ is the beamwidth for transmitters and by a factor of $2 \pi / \beta$, where $\beta$ is the beamwidth for receivers. Moreover, the results also indicate that if both transmitters and receivers employ directional antenna, the capacity can be improved by a factor of $4 \pi^{2} / \alpha . \beta$. In [11], Dai et. al extended the work on [7] on multi-channel multi-radio networks with the consideration of directional antennas.

Recent literatures indicate that employing capacity leverage unlimited bandwidth resources (UWB radios) can also improve the capacity bounds. Negi and Rajeswaran [9] show that when each node is constrained to a maximum transmit power $P_{0}$ and capable of utilizing $W \mathrm{~Hz}$ of bandwidth, the uniform throughput per node is upper bounded by $O\left((n \log n)^{(\alpha-1) / 2}\right)$ and lower bounded by $\Omega\left(\frac{n^{(\alpha-1) / 2}}{(\log n)^{(\alpha+1) / 2}}\right)$ under the limiting case when $B \rightarrow \infty$. In [12], the authors study the same problem in [9] and tighten both the lower and upper bounds of the network capacity to $\Theta\left(n^{(\alpha-1) / 2}\right)$ and close the gap between the upper and lower bounds that exist in [9].

Recently, power control in wireless networks has been the focus of extensive research. The main objectives of power control schemes is to reduce the total energy consumed in packet delivery and/or increase network throughput by increasing the channel's spatial reuse through the use of low transmission power in the network. In [13], the authors indicate that network capacity decreases significantly with higher transmission power and hence they suggest using the lowest transmission power to maximize the capacity of network. There are a lot of efforts following this suggestion [14],[15], and focus on using lower transmission power to maximize the network capacity. On the other hand, there is an opposite argument recently [16],[17]. In [17], Behzad et. al formulated the problem of power control as an optimization problem and showed that network capacity can be maximized by properly increasing the transmission power. Park et. al in [16] also showed through simulations that network capacity can occasionally increases with higher transmission power in certain scenarios. In [18], the authors analyzed why network capacity increases or decreases with higher transmission power in different scenarios, by using carrier sensing and minimum hop count in practice and showed that network capacity can be improved with higher transmission power in the networks with a small diameter.

\section{COnClusion}

In this paper, we have derived the lower and upper bounds on the capacity of MC-MR networks exploiting power in an arbitrary setting. Specifically, we answer the following questions: (a) Can we maximize the capacity of MC-MR wireless networks by increasing the transmission power?; (b) Under what criteria can we increase the transmission power of the nodes in a MC-MR network? The results derived in this paper indicate that it may be possible to build capacity-optimal wireless networks by exploiting the transmission power in MC-MR network under the following two constraints: (i) $c_{\text {min }}=O(c)$ and $c=O(n m)$; (ii) $c=\Omega(n m)$-where $c_{\text {min }}$ is the minimum number of channels required to achieve conflict-free transmissions in the network and $c$ is the number of existing channels. Furthermore, surprisingly our results also point out that if the maximum transmit power is $\Omega(n)$ i.e., $P_{\max }=\Omega(n)$, then partitioning the fixed bandwidth into $\Theta(\mathrm{nm})$ subchannels does not degrade the capacity of the wireless network which is an interesting and contrasting result to previously published works such as in [7]. In future, we also plan to study the capacity bounds of the proposed model under random placement of nodes. 


\section{APPENDIX}

While deriving the upper bounds for the MC-MR networks exploiting power in section VI, we assume the symmetric allocation model and hence the same transmit power level, $P\left(P_{0} \leq P \leq P_{\max }\right)$, for all nodes; Here, we adopt a different setting, termed as asymmetric allocation, than in section VI and analyze the upper bounds for capacity when $c_{\text {min }}<c<m n / 2^{5}$.

Theorem 5. Given $\gamma(0 \leq \gamma \leq 1)$, the transport capacity of the MC-MR network under asymmetric allocation in region $c_{\text {min }}<c<m n / 2$, measured in bit-meters/second, when node placements are chosen arbitrarily, in which the spatial reuse can be described by the sender-based interference model is:

$\frac{\sqrt{2} T}{(2+\Delta)}\left(k_{6} \gamma \frac{W}{c}+k_{5}(1-\gamma) \frac{W}{c}\left(\frac{c-\gamma c_{\min }}{(1-\gamma) c_{\min }}\right)\right) \sqrt{\frac{n m c_{\min }}{\pi}}$

Proof:

Let us suppose that $c$ channels are present in the network and no more than $c_{\min }$ channels are exploited. For a given parameter $\gamma(0 \leq \gamma \leq 1)$, we assume that interfaces tuned to $\gamma c_{\text {min }}$ channels transmit at power level $P_{0}$ and the remaining interfaces tuned to $(1-\gamma) c_{\min }$ can be tuned to a different power setting other than $P_{0}$. Then, we can interpret the following that since $c$ channels are present in the network, $c-\gamma c_{\min }$ channels can be divided among the interfaces tuned to $(1-\gamma) c_{\min }$ channels. Therefore to completely exploit the existing $c-\gamma c_{\min }$ channels, each node that is tuned to $(1-\gamma) c_{\text {min }}$ channels can increase the disk size by $\sqrt{\frac{c-\gamma c_{\min }}{(1-\gamma) c_{\text {min }}}}$. This in turn implies that each node can augment the power $P$ from $P_{0}$ to $P_{0}\left(\sqrt{\frac{c-\gamma c_{\min }}{(1-\gamma) c_{\min }}}\right)^{\alpha}$. Substituting $P_{0}\left(\sqrt{\frac{c-\gamma c_{\min }}{(1-\gamma) c_{\text {min }}}}\right)^{\alpha}$ in equation (4) and using Lemma 5 and $W_{x}=W / c$, we get $B=\frac{W}{c} k_{5} \frac{c-\gamma c_{\min }}{(1-\gamma) c_{\min }}$. Further, since each interface tuned to $\gamma c_{\text {min }}$ channels transmit at power level $P_{0}$, it transmits data at the rate of $B=\frac{W}{c} k_{6}$ bits per second. Now turn to the analysis of the upper bound. The difference stems from the need to replace (10) and (11) by different expressions. The expression in (10) and (11) are replaced by the following:

$H=\sum_{b=1}^{\lambda n T} h(b) \leq\left(k_{6} \gamma \frac{W}{c}+k_{5}(1-\gamma) \frac{W}{c}\left(\frac{c-\gamma c_{\min }}{(1-\gamma) c_{\min }}\right)\right) \frac{n m T}{2}$

$$
\begin{gathered}
\sum_{b=1}^{\lambda n T} \sum_{h=1}^{h(b)} \pi\left(1+\frac{\Delta}{2}\right)^{2}\left(d_{b}^{h}\right)^{2} \leq\left(k_{6} \gamma \frac{W}{c}+\right. \\
\left.k_{5}(1-\gamma) \frac{W}{c} \frac{\left(c-\gamma c_{\text {min }}\right)}{\left((1-\gamma) c_{\text {min }}\right)}\right) T c_{\text {min }}
\end{gathered}
$$

Following the same derivations in section VI, the capacity when $c_{\min }<c<m n / 2$ is upper bounded by

$$
\frac{\sqrt{2} T}{(2+\Delta)}\left(k_{6} \gamma \frac{W}{c}+k_{5}(1-\gamma) \frac{W}{c}\left(\frac{c-\gamma c_{\min }}{(1-\gamma) c_{\min }}\right)\right) \sqrt{\frac{n m c_{\min }}{\pi}}
$$

\footnotetext{
${ }^{5}$ the bounds remain the same for $c \geq m n / 2$ and $c_{\min }>c$ under both settings
}

Substituting $\gamma=0$ in (22) gives the transport capacity of the arbitrary network under asymmetric allocation as $\frac{\sqrt{2} k_{5} W T}{(2+\Delta)} \sqrt{\frac{n m}{\pi c_{\min }}}$ and setting $\gamma=1$ in (22) gives $\frac{\sqrt{2} k_{6} T}{(2+\Delta)} \frac{W}{c} \sqrt{\frac{n m c_{\min }}{\pi}}$ as the transport capacity which indeed is the capacity under power level $P_{0}$. Since the maximum network capacity is obtained when $\gamma=0$ (i.e., when all nodes employ same power $P>P_{0}$ ) we can clearly see that $\frac{\sqrt{2} k_{5} W T}{(2+\Delta)} \sqrt{\frac{n m}{\pi c_{m i n}}}$ is indeed the upper bound for the capacity when $c_{\min }<c<m n / 2$.

\section{REFERENCES}

[1] A. Kumar, D. Manjunath and J. Kuri. "Wireless Networking," in Morgan Kaufmann, pp. 1-448, 2008.

[2] P. Gupta and P. R. Kumar. "The capacity of wireless networks," IEEE Transactions and Information Theory, March 2000

[3] Yi Shi and Y. T. Hou. "Optimal Power Control for Multi-Hop Software Defined Radio Networks," in Proc. of IEEE Infocom, pp. 1694-1702, Anchorage, AK, May 2007.

[4] R. S. Diggavi, M. Grossglauser and D. Tse. "Even one-dimensional mobility increases adhoc wireless capacity," in Proc. of IEEE Int. Sump. Information Theory (ISIT), Lausanne, Switzerland, June 2002.

[5] M. Grossglauser and D. Tse. "Mobility increases the capacity of ad hoc wireless networks," in Proc. of IEEE Infocom, 2001.

[6] A. Gamal, J. Mammen, B. Prabhakar, and D. Shah "Throughput-delay trade-off in wireless networks," in Proc. of IEEE Infocom, 2002.

[7] P. Kyasanur and N.H. Vaidya, "Capacity of multi-channel wireless networks: impact of number of channels and interface," in Proc. of MOBICOM, pp. 43-57, Cologne, Germany, 2005.

[8] B. Liu, Z. Liu and D. Towsley "On the capacity of hybrid wireless networks," in Proc. of IEEE Infocom, 2003.

[9] R. Negi and A. Rajeswaran "Capacity of power-constrained ad hoc networks," in Proc. of IEEE Infocom, 2004.

[10] S. Yi, Y. Pei and S. Kalyanaraman "On the capacity improvement of ad hoc wireless networks using directional antenna," in Proc. of ACM Mobihoc, 2003

[11] H. Dai, K. Ng, R. Wong and M. Wu "On the Capacity of MultiChannel Wireless Networks Using Directional Antenna," in Proc. of IEEE Infocom, Phoenix, Arizona, April 13-18, 2008

[12] H. Zhang and J.C.Hou "Capacity of wireless ad-hoc networks under ultra wide band with power constraint," in Proc. of IEEE Infocom, 13-17 March 2005.

[13] S. Narayanaswamy, V. Kawadia, R.S. Sreenivas, and P. R. Kumar "Power Control in Ad hoc networks: Theory, Architecture, Algorithm and Implementation of the COMPOW protocol," in Proc. of European Wireless Conference, 2002.

[14] A. Muqattash and M. Krunz "Power Controlled Dual Channel (PCDC) Medium Access Protocol for Wireless Ad Hoc Networks," in Proc. of IEEE Infocom, vol. 1, pp. 470-480, 2003.

[15] - "A single-channel solution for Transmission power control in Wireless Ad hoc networks," in Proc. of IEEE Mobihoc, pp. 210-221, 2004.

[16] S. J. Park and R. Sivakumar "Load-sensitive Transmission Power control in Wireless Ad hoc networks," in Proc. of IEEE Globecom, vol. 1, pp. 42-46, 2002.

[17] A. Behzad and I. Rubin "High Transmission Power Increases the Capacity of Ad hoc wireless networks," IEEE Trans. on Wireless Communication, vol. 5(1), pp. 156-165, 2006.

[18] Y. Wang, J.C.S. Lui and D.Chiu "Understanding the paradoxical effects of power control on the capacity of wireless networks," IEEE Trans. on Wireless Communication, vol. 8(1), Jan, 2009.

[19] CellNode M100, www.sysmaster.com/brochures/cellnode-m100.pdf. 